\title{
Construction of a Quasi-Monoenergetic Neutron Source for Fast-Neutron Imaging
}

\author{
Johnson, Micah S. ${ }^{1,}{ }^{*}$, Anderson, Scott G. ${ }^{1}$, Bleuel, Darren L. ${ }^{1}$, Caggiano, \\ Joseph A. ${ }^{1}$, Fitsos, Peter, J. ${ }^{1}$, Gibson, David ${ }^{1}$, Gronberg, Jeff ${ }^{1}$, Hall, James M. ${ }^{1}$, \\ Marsh, Roark ${ }^{1}$, and Rusnak, Brian ${ }^{1}$ \\ ${ }^{1}$ Lawrence Livermore National Laboratory, 7000 East Ave. Livermore, California, 94551, USA \\ ajohnson329@IInl.gov
}

Keywords: Quasi-Monoenergetic Neutron Source, Fast-Neutron Imaging

\begin{abstract}
This paper presents and discusses an approach to fast-neutron imaging that will provide high-resolution detection (i.e. $\leq 1 \mathrm{~mm}$ ) of small features such as inclusions, voids, and variations in density. The application for fast-neutron imaging centers around assessing low-Z materials in high-Z shielded configurations. For this paper we present a simple theoretical argument on the feasibility of fast-neutron imaging and present results from some of our feasibility measurements. Finally, we discuss the requirements and objectives for the fastneutron imaging system currently under construction at Lawrence Livermore National Laboratory (LLNL).
\end{abstract}

\section{Introduction}

Neutron imaging was first demonstrated by Hartmut Kallmann and Ernst Kuhn ca. 1935 (c.f. [1]), shortly after the discovery of the neutron by Chadwick in 1932 [2]. Neutron radiography and x-ray radiography have mutually grown in parallel as technical analogs of each other and are complementary radiographic methods. X-rays and gamma-rays (i.e. high-energy $\mathrm{x}$-rays) interact with the electrons of an atom as well as the nucleus of an atom due to the electromagnetic properties of the photon. In general, this implies that photons interact (i.e. scatter) more readily with higher-Z materials. Neutrons, however, have no measurable net electric charge and can only interact with the nucleus of the atom via strong-nuclear interactions. The reduced interaction field allows the neutrons to penetrate further than photons, providing additional complementary depth to the probes of radiography. Reaction cross-sections for high-energy neutrons show only slight differences between many nuclei and can be represented by a simple model (see [3]). However, low-energy neutron reaction cross-sections vary considerably as a function of nuclei (c.f. [4]). These differences between photon- and neutron-reactions with atoms provide the underpinnings for the complementary nature of their respective radiographies.

Low-energy neutron radiography has been more extensively developed compared to highenergy neutron radiography because of the length scales of interest are on the order of crystalline lengths. (As a matter of reference, sample shapes have lengths approximately $29 \mathrm{fm} / \sqrt{ }$, where $T$ is the neutron kinetic energy in units of $\mathrm{MeV}$.) Additionally, it is easier to make low-energy neutron sources, especially with moderated fission reactors. Larger reaction cross-sections with different atomic nuclei also make low-energy neutron imaging particularly enticing. Low-energy neutrons can be used to measure the crystalline structure of solids using Bragg-edge scattering, which was developed by Enrico Fermi in 1947 [5]. Bragg-edges occur at neutron energies whose deBroglie wavelengths are consistent with twice the path-length difference from the lattice spacing as a function of incident angle. For lattice spacings on the order of an Angstrom, the neutron energies are about 1-10 meV. Examples of Bragg-edge scattering with low-energy 
neutrons can be found in [6,7], and references therein. Strain mapping such as tension and torsion can be accomplished with low-energy neutron diffraction. Position-sensitive detectors that surround a sample, irradiated by low-energy neutrons register diffraction peaks (c.f. [8]). Stress is then applied to the sample and the crystal dimensions change, resulting in a different diffraction pattern. Examples of strain mapping and other techniques can be found in [9,10], and references therein. Other techniques such as attenuation-based methods are used to test for porosity in materials.

High-energy neutrons ( $\geq 1 \mathrm{MeV}$ ) with $\sim$ fm-scale lengths do not have the right wavelengths necessary for applications presented above. Since fast neutrons cannot use the same techniques of diffraction, applications are limited. However, these fast neutrons have penetration power. With few exceptions, the fast-neutron total cross-sections are about 2-5 barns [3]. This crosssection range represents the lowest bounds of the slow-neutron total cross-section. (This is where the hard-sphere cross-section approximation of the nuclear size for low-energy neutrons transitions to neutron-nuclear interactions.) To first order, fast-neutron imaging can resolve mechanical defects of machined parts in heavily-shielded configurations. Fast-neutron imaging

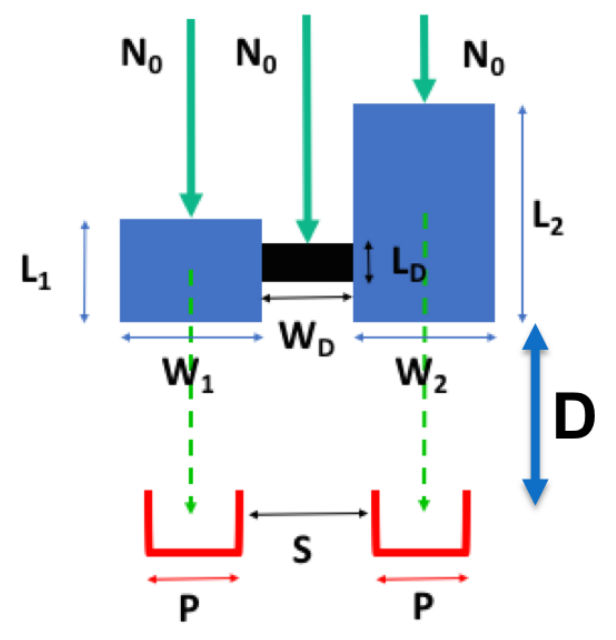

Figure 1: A simple model of a step-wedge with (lengths, widths) of $\left(L_{1}, w_{1}\right)$ and $\left(L_{2}\right.$, $\left.w_{2}\right)$. Also included, a test pillbox of (length, width) $\left(L_{D}, w_{D}\right)$. A flat distribution of neutrons is assumed and the distance from $a$ detector array is given to be $D$. The detector pixels are separated by a distance $s$ and each pixel width is $p$. A simple stepwedge estimate is obtained in the limit of $w_{D}$ goes to zero.

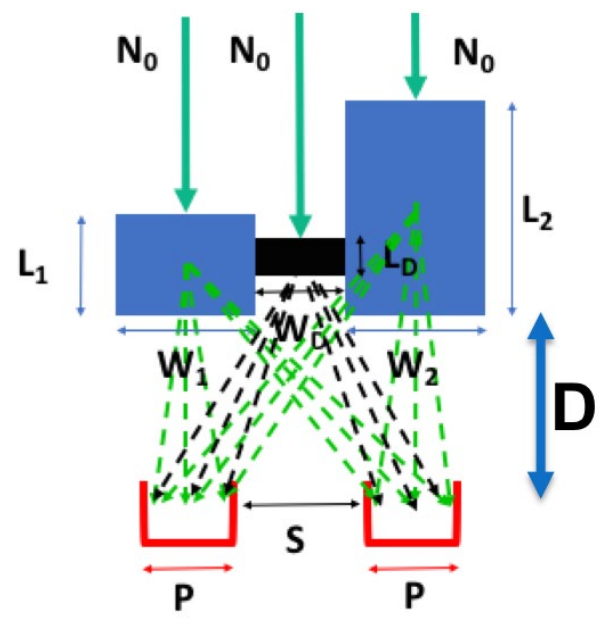

Figure 2: Same as Fig. 1, but with rays from the step-wedge components and test pillbox representing nuclear scatter. In practical applications, the step-wedge is surround by heavy shielding for which fast neutrons are known to penetrate.

can also resolve voids in a wide variety of materials in heavily-shielded containers. Fast-neutron imaging may have sensitivity of hydride and corrosion features in heavily-shielded scenarios. Regardless of the application objective, the value of fast-neutron imaging is dependent on its resolution. In the next section, we calculate the resolution of fast-neutron imaging with 
necessary input parameters. We then show results of our feasibility tests and discuss the design requirements of our fast-neutron imaging machine, which is under construction at LLNL.

\section{Model Estimates}

Putting aside any tangential benefits from fast-neutron radiography, our first objective is to be able to resolve and contrast a defect of interest against background with an analytical model. To do this, we use a simple step-wedge model in two dimensions with a test pillbox in between the two steps.

The analytical model we derive here is applicable to any imaging technique; the differences are in the input values for cross-sections and detector response. To begin, we define a six-step propagation (see Tab. 1) from the object plane (i.e. with the incident flux) to the digitizing plane assuming a lens-coupled CCD system.

Table 1: The (reduced) flux is described by $N$, with subscripts denoting the $n$-th step in the propagation series. The variance is denoted by $\sigma^{2}$. Attenuation through the material is denoted by $\tau$, which contains the relevant cross-sections. The other coefficients are efficiencies related to the detector. The values given are with respect to $10-\mathrm{MeV}$ neutrons for scintillator response and nominal values for the CCD and ADC collections and conversions.

- $N_{1}$ (object transmission $)=\tau N_{0}\left(\tau=f(E)\right.$; binomial) $\left(N_{0}\right.$ Poisson $)$

$-\sigma_{N_{1}}^{2}=N_{0} \sigma_{\tau}^{2}+\sigma_{N_{0}}^{2} \tau^{2}=N_{0} \tau(1-\tau)+N_{0} \tau^{2}=\tau N_{0}=N_{1}$ ( $\Rightarrow N_{1}$ Poisson $)$

- $N_{2}$ (scintillator reactions) $=\xi N_{1}(\xi=f(E)$; binomial)

$-\sigma_{N_{2}}^{2}=N_{1} \sigma_{\xi}^{2}+\sigma_{N_{1}}^{2} \xi^{2}=N_{1} \xi(1-\xi)+N_{1} \xi^{2}=\xi N_{1}=N_{2}$ ( $\Rightarrow N_{2}$ Poisson)

- $N_{3}$ (scintillator light yield $)=\delta N_{2}(\delta=f(E)$; Poisson $)$

$-\sigma_{N_{3}}^{2}=N_{2} \sigma_{\delta}^{2}+\sigma_{N_{2}}^{2} \delta^{2}=N_{2} \delta+N_{2} \delta^{2}=\delta N_{2}(1+\delta)=(1+\delta) N_{3}$ ( $\Rightarrow N_{3}$ non-Poisson)

- $N_{4}(\mathrm{CCD}$ light collection $)=\eta N_{3}(\eta \sim 2.822 \mathrm{E}-05$; binomial $)$

$-\sigma_{N_{4}}^{2}=N_{3} \sigma_{\eta}^{2}+\sigma_{N_{3}}^{2} \eta^{2}=\cdots=\eta N_{3}(1+\eta \delta)=(1+\eta \delta) N_{4} \quad\left(\Rightarrow N_{4}\right.$ non-Poisson $)$

- $N_{5}($ CCD electron yield $)=\varepsilon N_{4}(\varepsilon \sim 0.900$; binomial $)$

$-\sigma_{N_{5}}^{2}=N_{4} \sigma_{\varepsilon}^{2}+\sigma_{N_{4}}^{2} \varepsilon^{2}=\cdots=\varepsilon N_{4}(1+\varepsilon \eta \delta)=(1+\varepsilon \eta \delta) N_{5}$ ( $\Rightarrow N_{5}$ non-Poisson $)$

- $N_{6}$ (ADC count conversion $)=g N_{5}(g \sim 0.655 ;$ constant $)$

$-\sigma_{N_{6}}^{2}=N_{5} \sigma_{g}^{2}+\sigma_{N_{5}}^{2} g^{2}=\cdots=g N_{5} g(1+\varepsilon \eta \delta)=g(1+\varepsilon \eta \delta) N_{6}$ ( $\Rightarrow N_{6}$ non-Poisson $)$

In the context of our six-step model, contrast and fidelity is defined to be:

- $C \equiv \frac{\Delta N_{6}(\text { total })}{\sum N_{6}(\text { total })}$

- $F \equiv \frac{\Delta N_{6}(\text { total })}{\sigma_{N_{6}(\text { total })}}$,

where the $\Delta$-values are the differences in the final counts measured, the $\Sigma$-value is the total final counts, and $\sigma$ is the final count uncertainty. We parallelize our model into two scenarios where one source is assumed mono-energetic and the other to be a flat uniform spectral distribution, i.e. broadband uniform. Without too much difficulty, we derive an expression for contrast to be: 


$$
C=\tanh \Delta \gamma\left[\frac{1}{1+2 \frac{W_{D}}{W}\left(1+\frac{s}{p}\right) \frac{e^{-\Delta \phi} \cosh \Delta \gamma}{\cos }}\right],
$$

where:

- $\Delta \gamma \equiv \sqrt{2}\left(a_{2}-a_{1}\right)$;

- $\Delta \phi \equiv \sqrt{2}\left(a_{D}-\bar{a}\right)$;

- $a_{i} \equiv n_{i} \sigma_{i} L_{i}$

Here $n$ is the density of the component (see Figs. 1 and 2), $\sigma$ is the total cross-section (where the scattering component is distinct from 0-degrees), and $L$ are the component lengths, given in Figs. 1 and 2. One important feature for the contrast is that it is explicitly independent on spectral shape, i.e. broadband uniform or mono-energetic. The implicit dependence on spectral shape is in the cross-sections.

The fidelity can be shown to be:

$$
\begin{aligned}
& \text { - } F_{U}=\frac{2 E_{0} \sqrt{\Sigma N}\left(\sqrt{1+\varepsilon \eta \delta_{0}}-1\right)}{\sqrt{g} \varepsilon \eta \delta_{0}} \tanh \Delta \gamma\left[\frac{1}{1+2 \frac{W_{D}}{W}\left(1+\frac{s}{p}\right) \frac{e^{-\Delta \phi}}{\cosh \Delta \gamma}}\right] \\
& \text { - } \quad F_{M}=\frac{2 E_{0} \sqrt{\Sigma N}}{\sqrt{g} \sqrt{1+\varepsilon \eta \delta_{0}}} \tanh \Delta \gamma\left[\frac{1}{1+2 \frac{W_{D}}{W}\left(1+\frac{s}{p}\right) \frac{e^{-\Delta \phi}}{\cosh \Delta \gamma}}\right]
\end{aligned}
$$

Fidelity shows explicit spectral shape dependence. The ratio of these two is easily shown to be:

$$
\frac{F_{M}}{F_{U}}=\varepsilon \eta \delta_{0}\left[\frac{1}{1-\frac{1}{\sqrt{1+\varepsilon \eta \delta_{0}}}}\right]
$$

Since $\varepsilon \eta \delta_{0}>0$ (i.e. positive-definite); this implies that $\frac{F_{M}}{F_{U}}>1$, making mono-energetic sources the better fidelity. Resolution is defined to be: $R=\left|F_{\text {measured }}-F_{\text {expected }}\right|$. In the case of $W_{D} \sim W$ and $W_{D} \lesssim s, R=R_{G}(1+\tanh \Delta \gamma)$, where $R_{G}$ is the geometric (i.e. line-of-sight) resolution and is a function of step-width, pixel width, and pixel separation. For very small, $W_{D} \ll W, R=R_{G}\left(1+\frac{W_{D}}{W}\left(1+\frac{s}{p}\right) \frac{\tanh \Delta \gamma e^{-\Delta \phi}}{\cosh \Delta \gamma}\right)$, where it neatly reduces to line-of-sight resolution of a step-wedge if the width of the test pillbox is zero. Using the nominal values in Tab. 1 and average cross-sections for fast neutrons, and a signal-to-noise ratio of 5\%, and a flux on target of $10^{9}$ mono-energetic $10-\mathrm{MeV}$ neutrons, we estimate that the performance range is around $0.65-0.85 \mathrm{~mm}$. This range is subjective to uncertainties in the cross-sections and detector efficiencies. Moreover, this simplistic model does not consider scattering from shielding materials expected to be present, which is why we are considering fast neutrons. To perform more realistic calculations, we must use Monte-Carlo techniques to consider the scattering channels, especially from shielding.

Our realistic model is shown in Fig. 3. Figure 3 (Left) shows an Image Quality Indicator (IQI) consisting of concentric shells of high-Z and low-Z materials. The direction of the simulated beam is in the $+\mathrm{z}$ direction. Our results are based on analysis of a test pillbox (see Fig. 3 top), which is a volume, void of material. The pillboxes are placed at $(x, z)=(0,0)$ and at the $y-$ locations along the dashed lines shown in Fig. 3. The effective areal densities for the beam-path intersection of each pillbox are, $8.43 \mathrm{~g} / \mathrm{cm}^{2}, 137 \mathrm{~g} / \mathrm{cm}^{2}$, and $191 \mathrm{~g} / \mathrm{cm}^{2}$. The results of the analysis are given in table 2. 


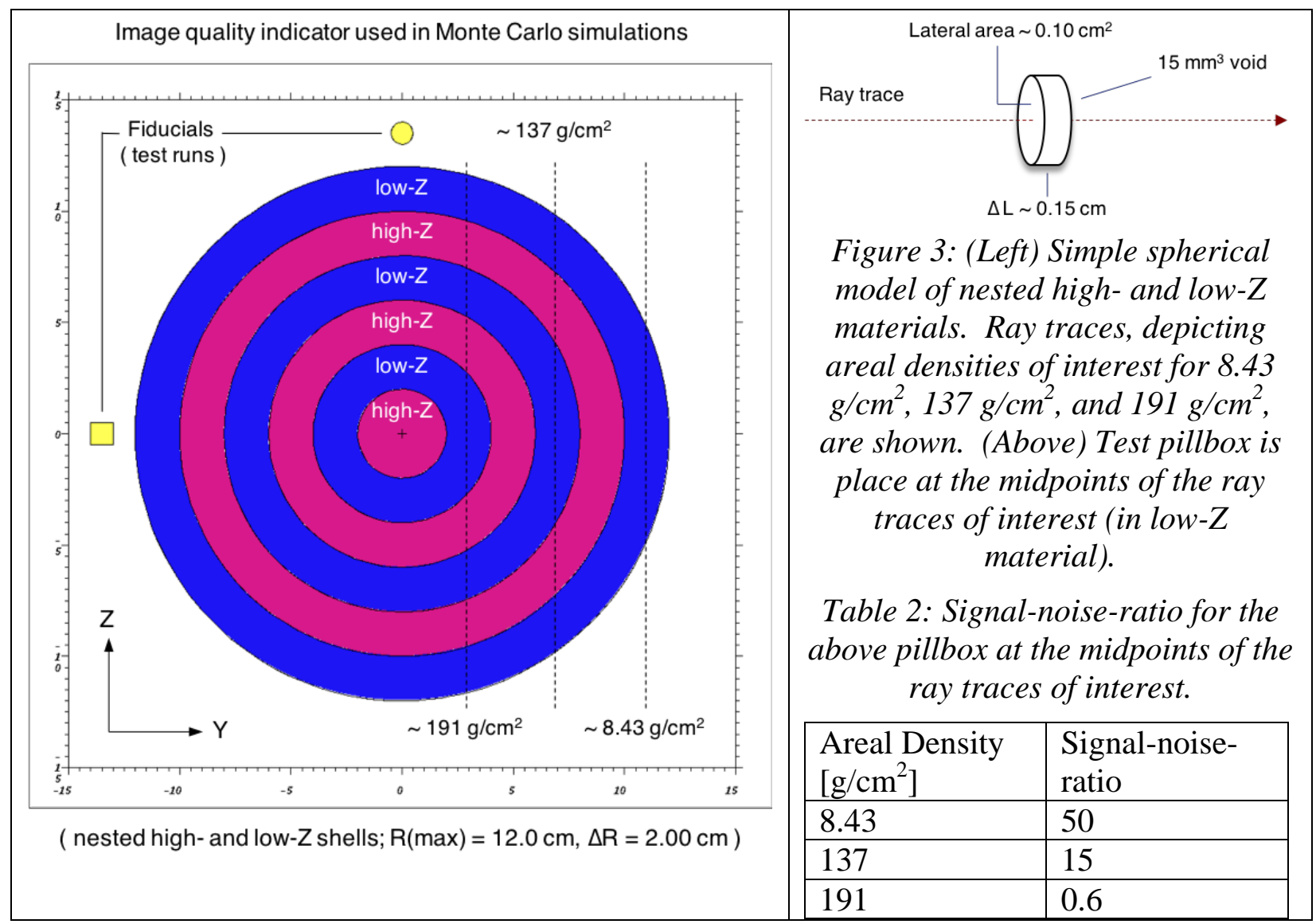

The results in Tab. 2 indicates good resolution for the areal densities denoted in Fig. 3 for the pillbox with a diameter of $\sim 3.7 \mathrm{~mm}$. It should be noted that the signal-noise-ratio for $137 \mathrm{~g} / \mathrm{cm}^{2}$, is approximately equivalent to $\mathrm{x}$-ray radiography using a $9-\mathrm{MeV}$ bremsstrahlung source. This represents the limit for high-energy x-ray radiography for test pillboxes depicted in Fig. 3.

\section{Feasibility Measurements}

Some of our feasibility measurements have been published by a number of us [11-16]. We report on one of our feasibility measurements that was performed with $10-\mathrm{MeV}$ quasimonoenergetic neutrons using the tandem Van De Graaf accelerator at the Edwards Accelerator Lab at Ohio University. 


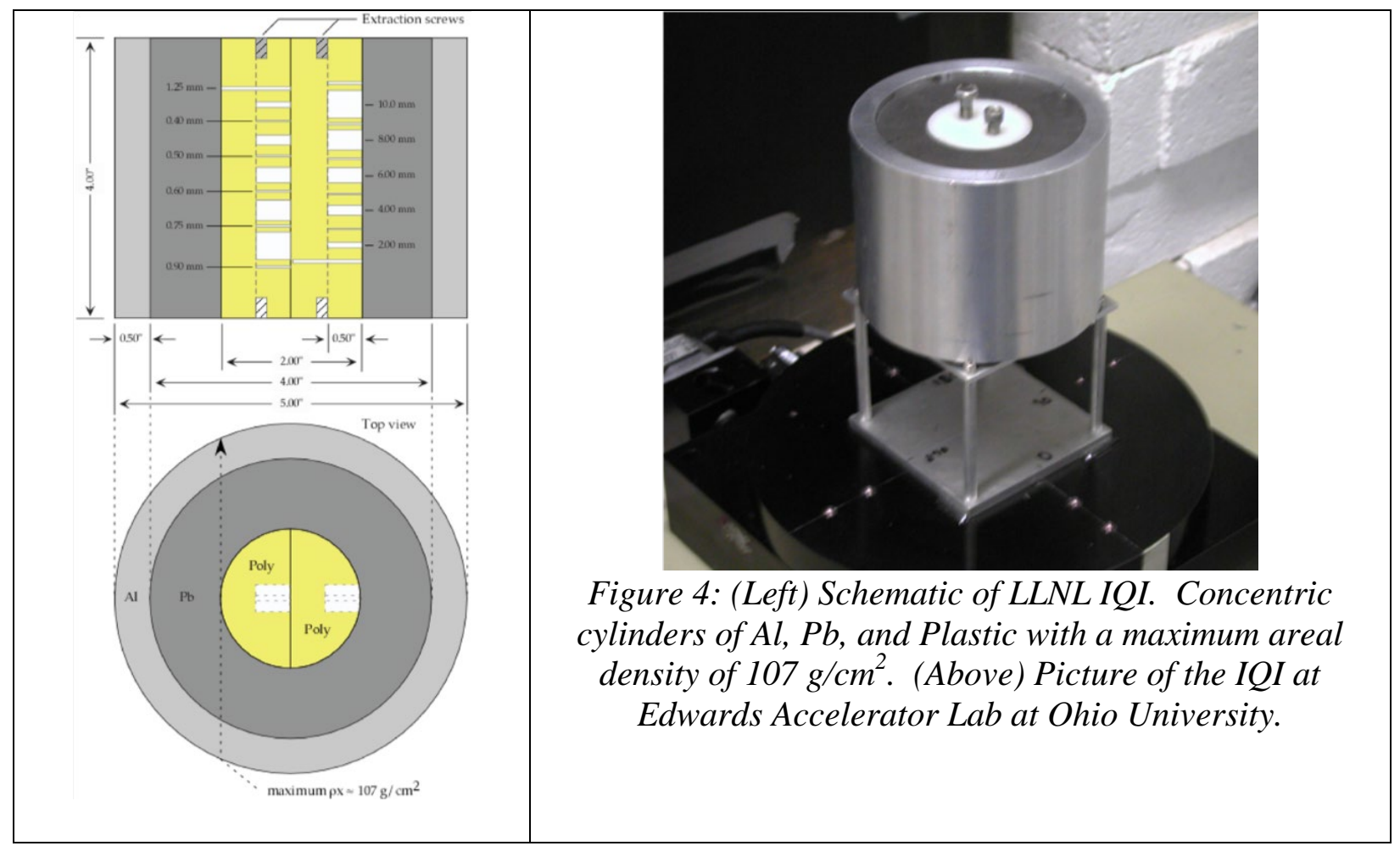

The IQI used is shown in Fig. 4. It is important to note that the maximum areal density is 107 $\mathrm{g} / \mathrm{cm}^{2}$. Our assessments, made for the above model (i.e. Fig. 3), is that the contrast for x-ray should be the same for $9-\mathrm{MeV}$ bremsstrahlung $\mathrm{x}$-rays and $10-\mathrm{MeV}$ neutrons. Our results are shown in Figs. 5 and 6. In both measurements, we used a lens-coupled CCD camera and used CT techniques with the same reconstruction methods.

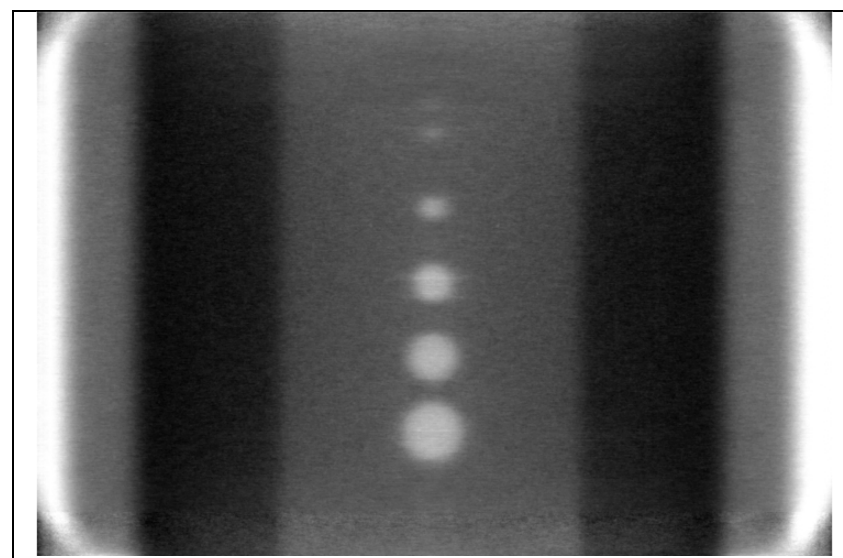

Figure 5: Neutron CT of IQI pictured in Fig. 4. The smallest test pillboxes in the plastic are clearly visible, and the contrast of the interface between the $\mathrm{Pb}$ and plastic is excellent.

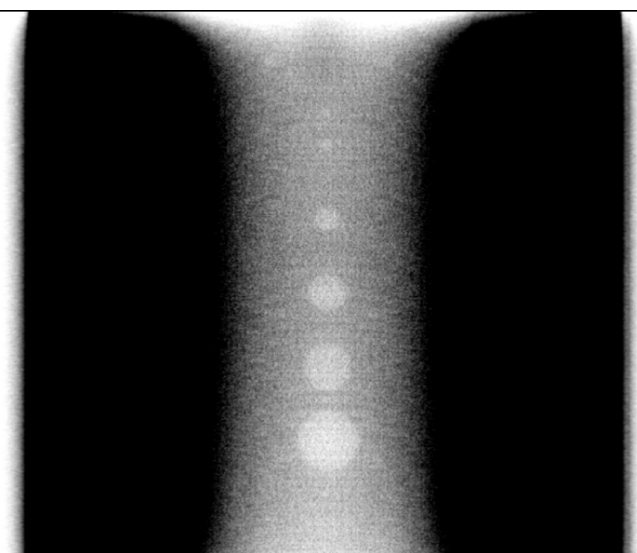

Figure 6: High-energy x-ray CT of same IQI in Figs. 4 and 5. Although the pillboxes are clearly visible, the contrast of the interface between the $\mathrm{Pb}$ and plastic is poor.

Although the test pillboxes are clearly visible in both the neutron- and x-ray-CT, the contrast between the low-Z and high-Z interface is poor for the $\mathrm{X}$-ray. Both of these observations are consistent with our calculations and estimates discussed above. 
In December 2017 we measured the above IQI, using a flat panel array, at the Los Alamos Neutron Science Center (LANSCE) at Los Alamos National Laboratory (LANL). LANSCE has a spallation source, which will provide a broadband flux. This data is still being analyzed.

\section{Construction of the LLNL source}

With the successes of our modelling and validation of our estimates, LLNL has begun construction on a 10-MeV quasi-monoenergetic neutron source at LLNL [17-28]. To meet demands of a high-brightness source, we are using a 300- $\mu \mathrm{A}, 100-\mathrm{Hz}$, deuteron accelerator, which consists of 2 radio frequency quadruoples (RFQ) and 1 drift tube linac (DTL). The deuterons are accelerated to $7 \mathrm{MeV}$ and impinge on a pulsed, windowless deuterium gas target system. (The necessity of the windowless gas target system is that the peak power on target is expected to be at $56 \mathrm{~kW}$ or $2.1 \mathrm{~kW}$ average.) The Q-value for $\mathrm{D}(\mathrm{d}, \mathrm{n})$ reaction then boosts the neutrons to $10-\mathrm{MeV}$. The length of the gas target is $4 \mathrm{~cm}$ and is maximum on the flight line at nearly 4 atm-gauge. The result is a neutron rate of $10^{11} \mathrm{n} / \mathrm{sr} / \mathrm{s}$ in the kinematically-focused forward cone of 10-degrees. The object- and image-plane will be adjustable between 1 and 5 meters for different magnifications. The source spot size (lateral area) will be around $10-\mathrm{mm}^{2}$, which is necessary for the sub-mm resolution in the object plane, we are striving for.

\section{Outlook}

Our plan for December 2018 is to measure the above IQI with filled-in features, using a flat panel array, at the Los Alamos Neutron Science Center (LANSCE) at Los Alamos National Laboratory (LANL). LANSCE has a spallation source, which will provide a broadband flux. We will then remeasure the IQI at LLNL's $10-\mathrm{MeV}$ quasi-monoenergetic source, currently under construction at LLNL. For the latter measurement we will use a flat panel array so that the nearly every aspect of the two measurements (LANL and LLNL) are equivalent with the exception of the source. This will validate and verify our assessments made above for broadband and monoenergetic effects on fidelity and resolution.

\section{Acknowledgements}

This work performed under the auspices of the U.S. Department of Energy by Lawrence Livermore National Laboratory under Contract DE-AC52-07NA27344.

\section{References}

[1] J. S. Brenzier, "A review of significant advances in neutron imaging from conception to the present”, Phys. Proc. 43 pp. 10-20 (2013). https://doi.org/10.1016/j.phpro.2013.03.002

[2] J. Chadwick, F. R. S., “The Existence of a Neutron”, Proc. R. Soc. Lond. A 136 pp. 692-708 (1932). https://doi.org/10.1098/rspa.1932.0112

[3] R.W. Bauer, J.D. Anderson, S.M. Grimes, and V.A. Madsen, “Application of simple Ramsauer model to neutron total cross sections”, UCRL-JC-127199 (1997)

[4] Hartnig, C. and Manke, I., "Neutron and synchrotron imaging, in-situ for water Visualization”, In Encyclopedia of Electrochemical Power Sources, Garche, J., et al. (Eds), Elsevier (2009), pp 738. https://doi.org/10.1016/B978-044452745-5.00078-2

[5] E. Fermi, W. J. Sturm, and R. G. Sachs, “The Transmission of Slow Neutrons through Microcrystalline Materials”, Phys. Rev. 71 n 9, pp. 589-594 (1947). https://doi.org/10.1103/PhysRev.71.589 
[6] W. Kockelmann, G. Frei, E.H. Lehmann, P. Vontobel, and J.R. Santisteban, “Energyselective neutron transmission imaging at a pulsed source”, Nucl. Instrum. And Meth. A 578 pp. 421-434 (2007). https://doi.org/10.1016/j.nima.2007.05.207

[7] Gian Song, et al., “Characterization of Crystallographic Structures Using Bragg-Edge Neutron Imaging at the Spallation Neutron Source”, J. Imaging 3, 65 (2017). https://doi.org/10.3390/jimaging3040065

[8] Winfried Kockelmann, et al., "Status of the neutron imaging and diffraction instrument IMAT”, Phys. Proc. 69 pp. $71-78$ (2015). https://doi.org/10.1016/j.phpro.2015.07.010

[9] Nikolay Kardjilov, Ingo Manke, André Hilger, Markus Strobl, and John Banhart, "Neutron imaging in materials science”, Materials Today Vol. 14 No. 6 (2011).

https://doi.org/10.1016/S1369-7021(11)70139-0

[10] Gian Song, et al., "Ferritic Alloys with Extreme Creep Resistance via Coherent Hierarchical Precipitates”, Sci. Rep. 5, 16327 (2015). https://doi.org/10.1038/srep16327

[11] F. Dietrich and J. Hall, "Report on measurements at Ohio University to estimate backgrounds for neutron radiography in the 10-14 MeV region”, UCRL-ID-127520 (LLNL, 1997). https://doi.org/10.2172/16133

[12] J. Hall, F. Dietrich, C. Logan, and B. Rusnak, "Recent results in the development of fast neutron imaging”, UCRL-JC-140345 (LLNL, 2000), Proc. $16^{\text {th }}$ Int. Conf. on the Applications of Accelerators in Research and Industry (Denton, TX, 2000), AIP CP576, 1113 (2001).

[13] J. Hall, “Uncovering hidden defects with neutrons”, Science \& Technology Review, UCRL52000-01-5 (LLNL, 2001)

[14] J. Hall, F. Dietrich, C. Logan, and B. Rusnak, "High-energy neutron imaging development at LLNL”, (LLNL, 2001), Proc. American Nuclear Society (2001).

[15] J. Hall, B. Rusnak, and P. Fitsos, "High-energy neutron imaging development at LLNL”, UCRL-CONF-230835 (LLNL, 2007), presented at Proc. $8^{\text {th }}$ World Conference on Neutron Radiography (Gaithersburg, MD, 2007). https://doi.org/10.2172/900879

[16] M. Johnson, S. Anderson, D. Bleuel, P. Fitsos, D. Gibson, J. Hall, R. Marsh, B. Rusnak, and J. Sain, "Development of a high-brightness, quasi-monoenergetic neutron source for neutron imaging”, Proc. Conference on the Application of Accelerators in Research and Industry (CAARI 2016). https://doi.org/10.1016/j.phpro.2017.09.018

[17] F. Dietrich and J. Hall, "Detector concept for neutron tomography in the $10-15 \mathrm{MeV}$ energy range”, UCRL-ID-123490 (LLNL, 1996). https://doi.org/10.2172/226435

[18] F. Dietrich, J. Hall, and C. Logan, "Conceptual design for a neutron imaging system for thick target analysis operating in the 10 - 15 MeV energy range”, UCRL-JC-124401 (LLNL, 1996), in Proc. $14^{\text {th }}$ Int. Conf. on the Applications of Accelerators in Research and Industry (Denton, TX, 1996), AIP CP392, 837 (1997). https://doi.org/10.1063/1.52470

[19] J. Hall, F. Dietrich, C. Logan, and G Schmid, "Development of high-energy neutron imaging for use in NDE applications,” UCRL-JC-134562 (LLNL, 1999), SPIE $\underline{3769}$, 31 (1999) and AIP 497, 693 (1999) (abridged version). 
[20] B. Rusnak and J. Hall, “An accelerator system for neutron radiography”, UCRL-JC-139558 (LLNL, 2000), Proc. $16^{\text {th }}$ Int. Conf. on the Applications of Accelerators in Research and Industry (Denton, TX, 2000), AIP CP576, 1105 (2001).

[21] B. Rusnak, J. Hall, and W. Hibbard, “A deuterium accelerator for neutron radiography”, UCRL-JC-145234 (LLNL, 2001), Proc. American Nuclear Society (Reno, NV, 2001). https://doi.org/10.1063/1.1395498

[22] B. Rusnak, J. Hall, and S. Shen, “A rotating aperture deuterium gas cell for high brightness neutron production”, UCRL-PROC-212293 (LLNL, 2005), Proc. 2005 Particle Accelerator Conference (Knoxville, TN, 2005)

[23] B. Rusnak, J. Hall, P. Fitsos, R. Souza, and M. Jong, “A large-format imaging optics system for fast neutron radiography”, UCRL-CONF-232018 (LLNL, 2007), in Proc. 2007 Particle Accelerator Conference (Albuquerque, NM, 2007). https://doi.org/10.1109/PAC.2007.4440657

[24] P. Fitsos, S. Edson, J. Hall, and B. Rusnak, “Design and fabrication of a precision pulsed valve system for neutron imaging”, LLNL-CONF-491678 (LLNL, 2011), Proc. 26th Annual Meeting of the American Society for Precision Engineering (ASPE) (Denver, CO, 11/11).

[25] V. Tang, B. Rusnak, S. Falabella, J. McCarrick, H. Wang, J. Hall, and J. Ellsworth, “Fusiondriven gamma and fast neutron radiography test-bed at Lawrence Livermore National Laboratory”, Proc. $22^{\text {nd }}$ Int. Conference on Application of Accelerators in Research and Industry (CAARI) (Fort Worth, TX, 08/12).

[26] B. Rusnak, J. Hall, P. Fitsos, M. Johnson, D. Bleuel, A. Weidrick, M. Crank, S. Anderson, R. Marsh, D. Gibson, J. Sain, and R. Souza, "Development of a high-brightness source for fast neutron imaging”, Proc. 2013 North American Particle Accelerator Conference (Chicago, IL, 10/16)

[27] B. Rusnak, J. Hall, P. Fitsos, M. Johnson, D. Bleuel, M. Crank, S. Anderson, R. Marsh, D. Gibson, J. Sain, L. Kruse, G. Anderson, S. Fisher, D. Nielsen, K. Lange, D. Jamero, and O. Alford, “Advancement of an accelerator-driven high-brightness source for fast neutron imaging”, Proc. of 2017 International Conference on Particle Accelerators (Copenhagen, Denmark, 05/17).

[28] R. Marsh, G. Anderson, S. Anderson, D. Bleuel, M. Crank, P. Fitsos, D. Gibson, J. Hall, M. Johnson, B. Rusnak, J. Sain, R. Souza, and A. Wiedrick, "High average power deuteron beam dynamics”, Proc. of 2017 International Conference on Particle Accelerators (Copenhagen, Denmark, 05/17). 